\title{
Correction to: Immunosuppression, Including Drug Toxicity, Interactions, New Immunosuppressants in the Pipeline
}

Denise Wang, Bruno Meiser, Howard J. Eisen, and Sandra Eifert

\section{Correction to:}

Chapter 19 in: D. S. Feldman, P. Mohacsi (eds.), Heart Failure, Cardiovascular Medicine, https://doi.org/10.1007/978-3-319-98184-0_19

The original version of this chapter was revised. This chapter was inadvertently published with incorrect author name "Denise Wanga" instead of "Denise Wang". 